\title{
THREE-DIMENSIONAL MR ANGIOGRAPHY FOR PLANNING OF HEPATIC ARTERIAL CATHETERIZATION
}

\author{
Yoshinori Koyama ${ }^{1)}$, Tomio Inoue ${ }^{1)}$, Hideyuki Ishijima ${ }^{1)}$, \\ Jun Aoki ${ }^{2)}$, Keigo Endo ${ }^{1)}$ \\ From the Department of Nuclear Medicine ${ }^{1)}$ and Diagnostic Radiology ${ }^{2)}$, \\ Gunma University School of Medicine, 3-39-22, Showa-machi, Maebashi, Gunma, 371-8511, Japan
}

\begin{abstract}
The aim of this study is to assess the potential utility of gadolinium-enhanced threedimensional MR angiography (3D MR angiography) for arterial catheterization of hepatic tumor. Thirty-five consecutive patients with suspected abdominal tumors underwent MR angiography with a gadolinium-enhanced 3D fast gradient echo sequence. Visualization of the vascular tree of visceral arteries, and their variations, the apparent length of common hepatic artery (CHA), and the tilt of celiac trunk were prospectively evaluated by 3D MR angiography. The results were compared with those obtained by the conventional angiography. In 27 patients with hepatic tumors, the prospective planning with 3D MR angiography was compared with the actual catheterization. Celiac trunk, CHA, and superior mesenteric artery (SMA) were visualized on 3D MR angiography in all 35 patients. There was a significant linear correlation of the length of CHA and the tilt of SMA as measured by 3D MR angiography and conventional angiography $(r=0.75, r=0.64$, respectively). The 3D MR angiography provided useful clinical information for planning of arterial catheterization in all 27 patients with hepatic tumors. Thus gadolinium-enhanced 3D MR angiography is considered to be of value for the planning of arterial catheterization of hepatic tumor.
\end{abstract}

Key words: Magnetic resonance, Vascular studies, Angiography, Liver neoplasm, Interventional procedure

(Kitakanto Med.J. 50 (2) : 93 98, 2000)

\section{INTRODUCTION}

Interventional radiology is a rapidly developing subspecialty in treatment of patients suffering from malignant tumors, vascular diseases and massive bleeding. Its utility depends primarily on the development of various devices such as catheters, coils and stents, which require precise planning based on vascular anatomy prior to insertion. Information of vascular anatomy has been mostly obtained by conventional angiography performed on previous days or just prior to the intervention. The drawbacks of this method include invasiveness and limited time for the discussion of procedure. Since we have experienced technological difficulties due to the unusual vascular anom- aly, there is a clinical advantage of pretreatment planning of intervention using non-invasive imaging modalities. Some non-invasive imaging modalities providing the information about vascular anatomy include: ultrasonography (US), enhanced computer tomography (CT), magnetic resonance (MR) imaging by time of flight (TOF) method. Although MR imaging of two-dimensional (2D) TOF is preferable technology, it is time-consuming and provides poor image quality $^{1,2)}$. The technology of gadolinium-enhanced three-dimensional magnetic resonance angiography (3D MR angiography) was proposed and used in clinical practice ${ }^{3 \sim 5}$. Based on the clinical study, gadolinium-enhanced 3D MR angiography has been proved to be a useful technique for the evaluation of 
vascular stenosis of renal arteries, iliac arteries ${ }^{6)}$, and superior mesenteric artery (SMA) $)^{7}$. However, there are few reports describing its application for planning of interventional procedure ${ }^{8}$. The aim of this study is to assess the potential utility of gadolinium-enhanced 3D MR angiography for the planning of arterial catheterization of hepatic tumor.

\section{SUBJECTS AND METHODS}

Thirty-five consecutive patients who underwent 3D MR angiography prior to conventional abdominal angiography were involved in this study. Twenty-five patients had hepatocellular carcinoma (HCC), 5 had metastatic hepatic tumor, and 5 had pancreatic tumor. Subjects in this study were 23 males and 12 females whose age ranged from 46 to 77 (mean age $65 \mathrm{yr}$.). Diagnostic confirmation of HCC, metastatic hepatic cancer, or pancreatic cancer in 27 patients was based on pathological examination by ultrasound-guided biopsy or operation. The diagnoses in 8 patients were based on findings of imaging modalities of US, CT, and angiography, the results of clinical laboratory tests including tumor markers, and clinical observation of patients. After 3D MR angiography, 27 patients underwent vascular interventional treatment; 24 patients with $\mathrm{HCC}$ and 1 patient with metastatic hepatic cancer underwent transcatheter arterial embolization (TAE), 1 patient with metastatic hepatic cancer underwent percutaneous reservoir catheter placement by femoral catheterization and 1 patient with metastatic hepatic cancer underwent transcatheter arterial infusion (TAI).

\section{$M R A$}

MR examinations were performed using a $1.5-\mathrm{T}$ system (Signa Horizon; GE Medical System, Milwaukee, WI) equipped with a high-performance three-axis gradient configuration characterized by maximum amplitude of $23 \mathrm{mT} / \mathrm{m}$ and a slew rate of $77 \mathrm{mT} / \mathrm{m} /$ msec. The pre-contrast axial MR images were acquired for positioning the visceral artery of 3D MR angiography: T1-weighted spin echo images $(400-450 / 8$ [repetition time msec/echo time $\mathrm{msec}], 7-\mathrm{mm}$ slice thickness, echo train $12,512 \times 192$ matrix, two signals acquired, superior and inferior saturation) and fast spin-echo T2-weighted images (4000-6000/8[respiration triggered]/102[effective], 7-mm slice thickness, echo train $12,512 \times 192$ matrix, two signals acquired).

After acquisition of pre-contrast axial $\mathrm{T} 1$-and T2-weighted images, breath-hold 3D MR angiography was conducted. The field of view (FOV) of 3D MR angiography was determined by the location of celiac trunk and main lesion on the pre-contrast axial MR images. 3D MR angiography was obtained with an enhanced 3D fast gradient echo sequence with 32 sections in the coronal plane $\left(6.2 / 1.4,30^{\circ}\right.$ flip angle, $32-\mathrm{cm}$ FOV, $32-\mathrm{kHz}$ bandwidth, $256 \times 128$ matrix, slice 5 -mm thickness, one signal acquired). This sequence was repeated four times; pre-contrast, arterial phase, portal phase, and delayed phase. Each patient held his/her breath for 22 seconds during each imaging. A 22 gauge intravenous catheter was placed in an antecubital vein and a $15 \mathrm{ml}-G a d o l i n i u m ~ d i e t h-$ yltriaminepentaacetic acid (Gd-DTPA) was injected at a rate of $1 \mathrm{ml} / \mathrm{sec}$ followed by infusion of $10 \mathrm{ml}$ saline. MR angiography of arterial phase was initiated 15 seconds after the initiation of contrast media administration. Imaging of portal phase and delayed phase was also initiated 13 seconds, and 97 seconds, respectively, after the completion of arterial phase imaging.

\section{Angiography}

Conventional angiography using the system of digital subtraction angiography (DSA) (Digitron 3 and Multistar T.O.P./Plus, Siemens, Erlangen, Germany) was performed as the standard of reference in all patients after the completion of 3D MR angiography. A femoral approach was conducted with a 5-F catheter positioned at celiac trunk and SMA, with injection of $20-30 \mathrm{ml}$ iomeprol at a rate of $5-8 \mathrm{ml} / \mathrm{sec}$. Anteroposterior view angiograms were obtained in all patients. In 10 patients, lateral view aortograms were added for the measurement of tilt degree of celiac trunk and SMA. Microcatheter was used for selective angiography, TAE and TAI.

\section{Image Interpretation and Comparison with the Stan- dard Reference}

MR images were evaluated by two experienced vascular intervention radiologists (Y.K., H.I.), using a workstation (Advantage Windows; GE Medical System). Maximum intensity projection (MIP) and multiprojection volume reconstruction (MPVR) images were generated in the coronal images through celiac and SMA. The source images were not employed in this evaluation.

Visualization of the vascular tree of visceral arteries and its variations on 3D MR angiography was prospectively interpreted for the following segments : celiac trunk, common hepatic artery (CHA), splenic artery, left gastric artery, gastroduodenal artery, proper hepatic artery, right and left hepatic artery, and SMA. Tilting pattern of celiac trunk in respect to the anterior-posterior perpendicular to the body axis was classified as upward and downward on 3D MR angiography and conventional angiography. In cases where visual interpretation of 3D MR angiography yielded 
different results, final results were obtained by consensus of two vascular intervention radiologists.

Quantitative interpretation of 3D MR angiography was conducted on the apparent length of $\mathrm{CHA}$ on antero-posterior view image. The apparent length of CHA was defined as the summation of the linear length from the proximal end of $\mathrm{CHA}$ to the mid-point of $\mathrm{CHA}$ and that from the peripheral end of $\mathrm{CHA}$ to the mid-point of CHA. The tilt degree of celiac trunk or SMA on the lateral view was determined by defining two lines along the abdominal aorta and celiac trunk or SMA. All visual and quantitative interpretations of 3D MR angiography were compared with the results obtained through conventional angiography.

\section{Planning of hepatic arterial catheterization based on findings of 3D MR angiography}

In 27 patients who underwent interventional procedure for hepatic cancer, the planning of hepatic arterial catheterization was conducted prospectively based on findings of 3D MR angiography. For the preparation of catheter, the tilt of celiac trunk on the lateral view was categorized as follows: group 1:<90 degrees, group 2: 90-135 degrees, group $3: 135$ degrees $<$.

Determination of catheter was performed by the actual intervention. Prospective planning of arterial catheterization with 3D MR angiography was compared with the actual results.

\section{Statistical Analysis}

The relationship between measurement of the apparent length of CHA, the tilt of celiac trunk and SMA by 3D MR angiography and conventional angiography was determined by linear regression analysis. The agreement of the tilting pattern of celiac trunk was estimated by the Cohen $\varkappa$ statistics $^{9,10)}$. The values ranged from -1 (no agreement) to 1 (perfect agreement), and the agreement was classified as poor $(=0.00)$, slight (0.00-0.20), fair (0.21-0.40), moderate (0.41-0. $60)$, substantial (0.61-0.80), or almost perfect (0.81 $-1.00)^{11}$.

\section{RESULTS}

Celiac trunk, CHA, and SMA were visualized on 3D MR angiography in all 35 patients. Proper hepatic artery was not visualized in one case. Eight of 35 patients revealed the difficulty of identification of left gastric artery (Table 1). Three patients showed branch variation of visceral arteries; two with right hepatic artery branched from SMA (replaced right hepatic artery), one with left hepatic artery branched from left gastric artery (replaced left hepatic artery) and right hepatic artery branched from SMA (replaced right
Table 1 Identification of Visceral Arteries on 3D MR Angiography

\begin{tabular}{lc}
\hline \multicolumn{1}{c}{ Visceral arteries } & $\begin{array}{c}\text { Number of visualized arteries } \\
(\mathrm{n}=35)\end{array}$ \\
\hline Celiac trunk & $35(100)$ \\
Common hepatic artery & $35(100)$ \\
Proper hepatic artery & $34 \quad(97)$ \\
Right hepatic artery & $30(85.7)$ \\
Left hepatic artery & $28 \quad(80)$ \\
Gastroduodenal artery & $31(88.6)$ \\
Splenic artery & $34 \quad(97)$ \\
Left gastric artery & $27 \quad(77)$ \\
Superior mesenteric artery & $35 \quad(100)$ \\
\hline
\end{tabular}

*Numbers in parenthesis indicate percentage (\%) compared with the conventional angiography as the standard reference.

(a)

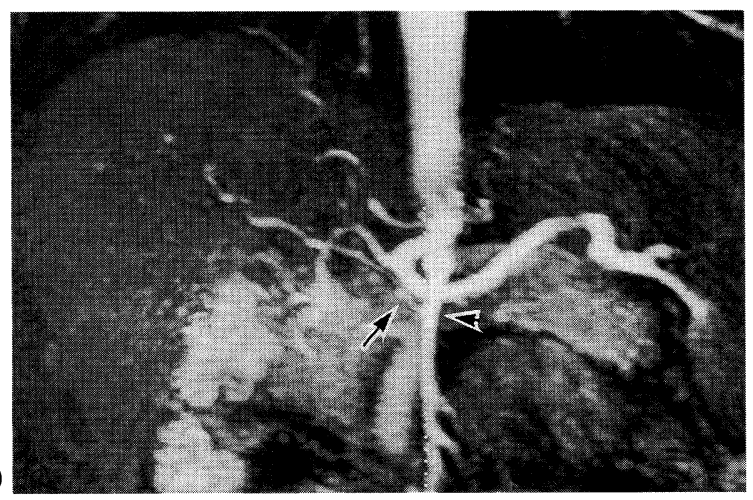

(b)

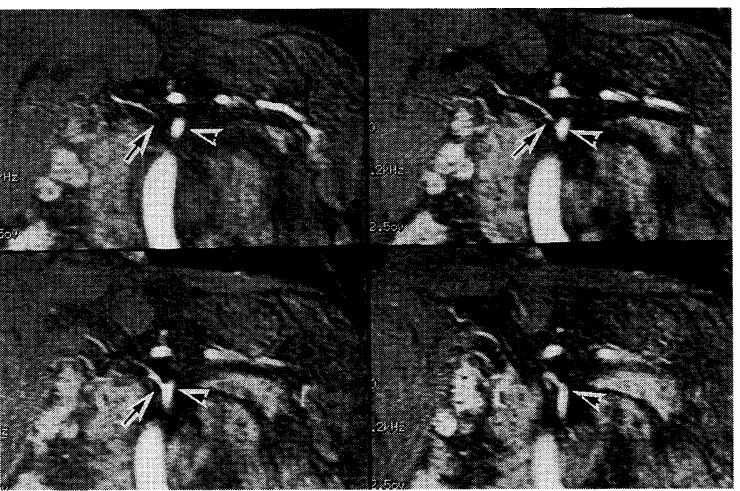

(c)

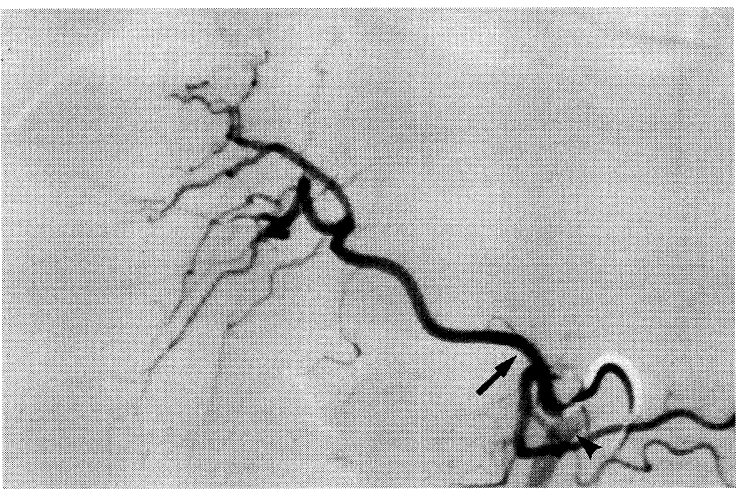

Fig. 1. Replaced right hepatic artery in a 69-year-old male patient with hepatocellular carcinoma. 3D MR angiography of multi-projection volume reconstruction (MPVR) image (a) revealed a replaced right hepatic artery (arrow) branched from superior mesenteric artery (arrowhead), which was confirmed on source images of MR angiography (b) and conventional angiography (c). 
Table 2 Comparison of the Evaluation of Celiac Trunk Tilt

\begin{tabular}{|c|c|c|}
\hline MR Angiography & Conventional Angiography & Number of patients(\%) \\
\hline $\mathrm{U}$ & U & $20^{*}(57.1)$ \\
\hline $\mathrm{D}$ & $\mathrm{D}$ & $5^{*}(14.3)$ \\
\hline $\mathrm{U}$ & $\mathrm{D}$ & $3 \quad(8.6)$ \\
\hline D & $\mathrm{U}$ & (20) \\
\hline
\end{tabular}

$\mathrm{U}=$ upward, $\mathrm{D}=$ downward

${ }^{*}$ Cohen $\varkappa$ statistics ; $\varkappa=0.31$; The agreement was fair.

(a)

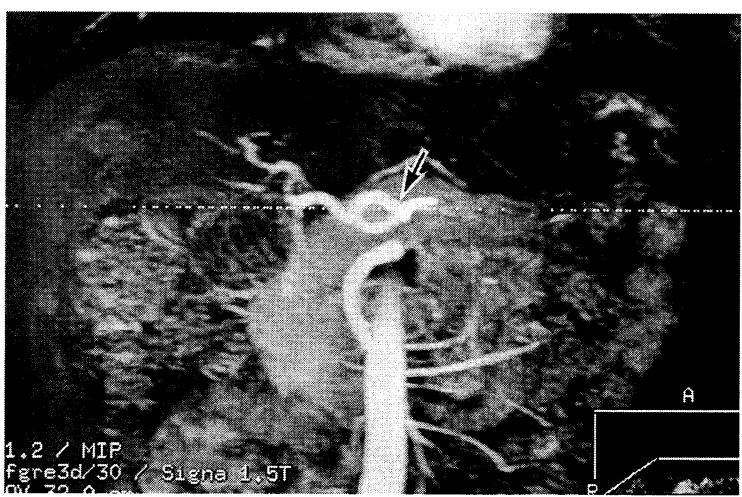

(b)

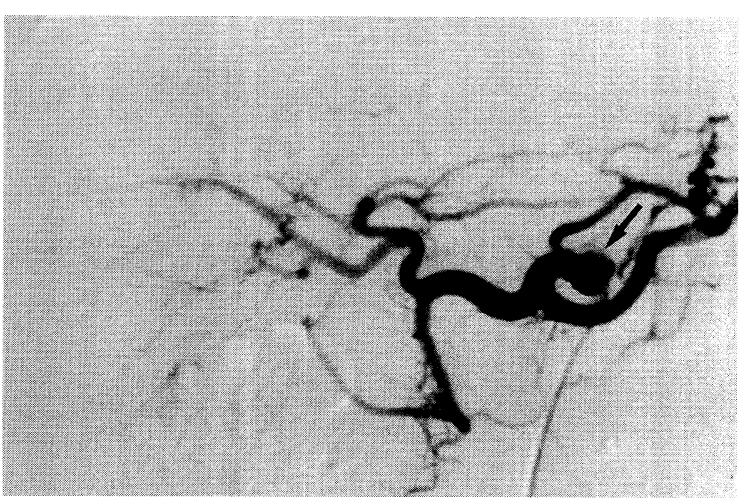

Fig. 2. Evaluation of the tilt of celiac trunk in a 59-year-old male patient with metastatic liver tumors from rectal cancer. The tilting pattern of celiac trunk was evaluated as upward tilt (arrow) both on 3D MR angiography of multiprojection volume reconstruction (MPVR) image (a) and conventional angiography (b).

hepatic artery). All variations in these three patients were prospectively accurately recognized on $3 \mathrm{D}$ MR angiography prior to TAE (Fig 1a, 1b, 1c).

Twenty-seven cases of upward tilting and 8 of downward tilting of celiac trunk were recognized on conventional angiography. The evaluations of 3D MR angiography regarding tilting pattern of celiac trunk coincided in 20 patients with upward tilting (Fig 2a, $2 \mathrm{~b}$ ), and in 5 patients with downward tilting. Ten of 35 patients $(28.6 \%)$ showed inconsistent results of evaluated tilting pattern of celiac trunk (Table 2). The agreement of the tilting pattern of celiac trunk was estimated as a fair agreement $(\varkappa=0.31)$ by the Cohen $\varkappa$ statistics.

There was a significant linear correlation between

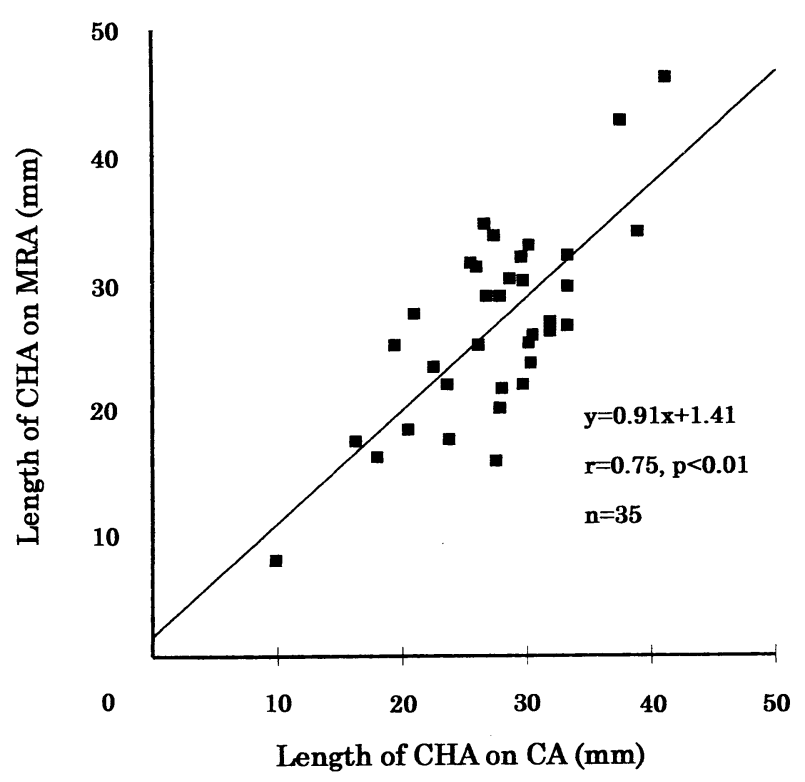

Fig. 3. Relationship of the length of common hepatic artery (CHA) as measured by 3D MR angiography (MRA) and by conventional angiography (CA). There was significant linear correlation between the two $(r=0.75, n=35$, $\mathrm{p}<0.01)$.

the length of $\mathrm{CHA}$ as measured by 3D MR angiography and by conventional angiography $(r=0.75, n=35$, $\mathrm{p}<0.01$ ) (Fig 3). Although there was a significant linear correlation between the measurement of the tilt of SMA produced by 3D MR angiography and by conventional angiography $(\mathrm{r}=0.64, \mathrm{n}=10, \mathrm{p}<0.05)$, the correlation in the case of the tilt of celiac trunk was not significant $(\mathrm{r}=0.39, \mathrm{n}=10$, n.s.) (Fig 4a, 4b).

There were 5 patients of group 1, 21 patients of group 2 , and 1 patient of group 3 . In the case of group 1 , an obtuse-angled catheter such as cobra catheter and twisted-shaped catheter was suitable. In the case of group 2, either obtuse-angled or acute-angled catheters were required. In the case of group 3 , an acute-angled catheter such as 'shepherd hook' catheter was recommended. Catheters selected on the basis of the degree of tilt of celiac trunk by 3D MR angiography were not changed during the actual interventional procedure. With respect to the planning of arterial catheterization, the catheter selection based on the findings of 3D MR angiography coincided well with the actual procedures.

\section{DISCUSSION}

In planning the interventional procedure of visceral artery, anatomical information of main branches, such as celiac trunk, CHA, gastroduodenal artery, and SMA, is important for ensuring a successful treatment, shortening the time of procedure, and preventing complications ${ }^{12}$. In our study, 3D MR angiography pro- 


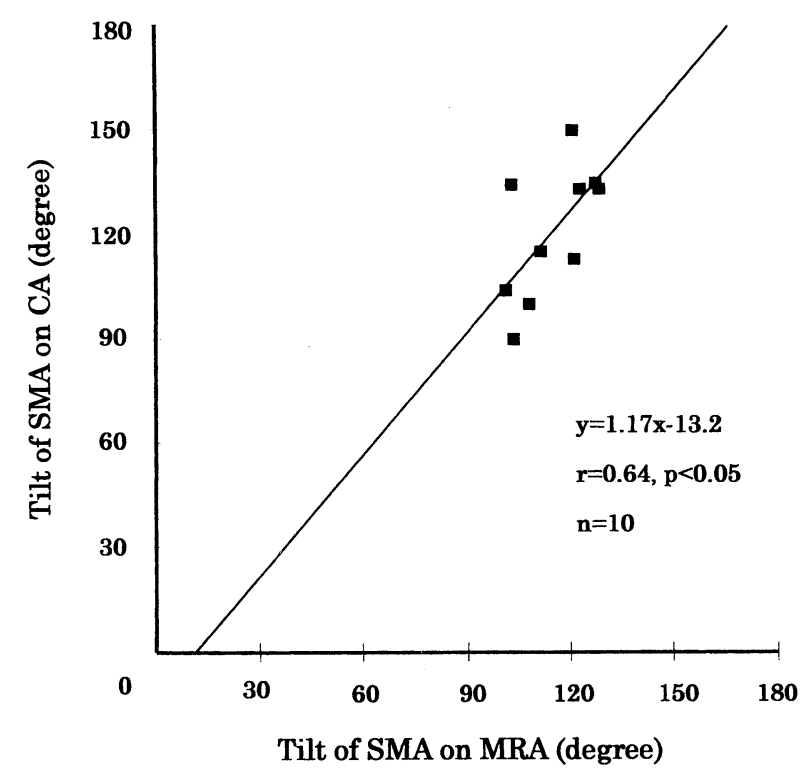

(a)

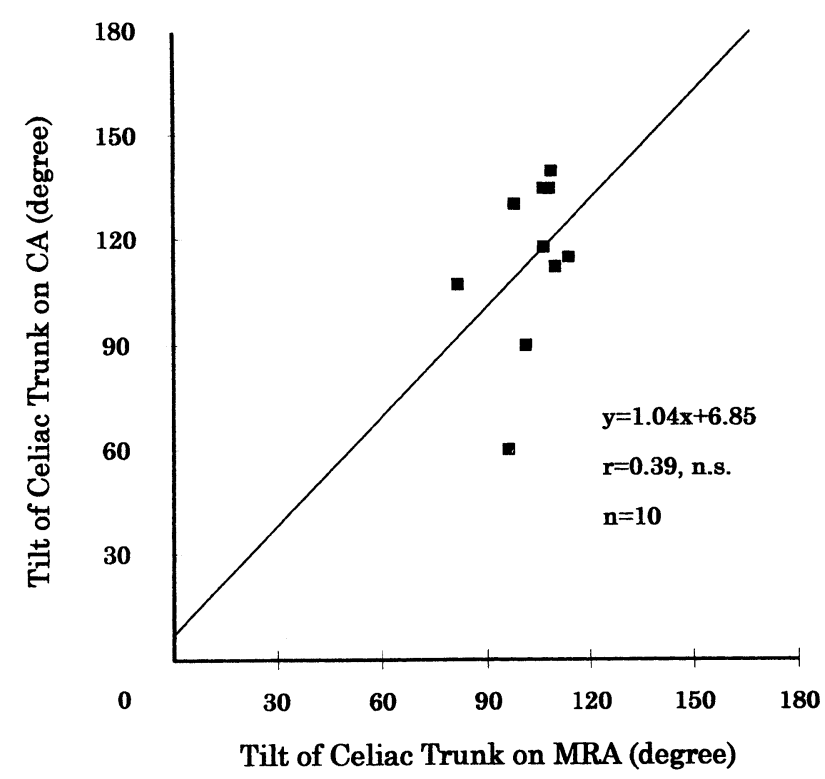

(b)

Fig. 4. Relationship of the tilt of superior mesenteric artery (SMA) (a) and celiac trunk (b) as measured by 3D MR angiography and conventional angiography (CA). Although there was significant linear correlation of the tilt of SMA $(r=0.64, n=10, p<0.05)$, there was no significant correlation of the tilt of celiac trunk $(r=0.39, n=10$, n.s. $)$.

vided anatomical information of main branches of visceral arteries (Table 1). Prior information of branch variations helped us to perform an efficient TAE or percutaneous reservoir catheter placement by femoral catheterization. In the case of patients with replaced right or left hepatic artery, additional microcatheters and/or metalic coils would be necessary for the selective insertion of $\mathrm{TAE}^{13}$ and arterial redistribution of percutaneous reservoir catheter placement ${ }^{14 \sim 16)}$. Although branch variations may be identified by using the transaxial images of CT and MRI, 3D MR angiography can provide more precise information. 3D MR angiography also provided accurate information of branch variations in all three patients (Fig 1). Although the information of peripheral branch anatomy such as segment branch of hepatic artery, right gastric artery, pancreaticoduonenal artery, and dorsal pancreatic artery is also important for successful intervention, it seemed to be difficult to obtain the information using 3D MR angiography of MIP and MPVR images because of its limitation of spatial resolution.

If the tilting pattern of celiac trunk can be evaluated prior to the interventional procedure, proper catheter can be easily prepared. Regarding the tilting pattern of celiac trunk, the results obtained by 3D MR angiography and conventional angiography proved to be inconsistent in 10 of 35 (28.6\%) patients (Table 2). It may be due to the inconsistency of breath hold position since the celiac trunks of these patients did not display an extremely acute angle. Even if any type of catheter such as cobra, twisted-shaped and "shep- herd hook" were suitable for most patients, catheter exchange during catheterization was needed in some patients with an extremely acute or obtuse angle of celiac trunk ${ }^{17)}$. Appropriate selection of the catheter that fits the vascular anatomy has produced a 95\% success rate in the hepatic artery catheterization ${ }^{17)}$. In this study, an approximate classification in 3 groups of tilt of celiac trunk was available for the catheter selection.

The information regarding the length of $\mathrm{CHA}$ is important to decide the position of percutaneous reservoir catheter placement ${ }^{15,16)}$. A significant correlation between the measurements of the apparent length of CHA by 3D MR angiography and conventional angiography was confirmed in this study (Fig 3).

In conclusion, 3D MR angiography may provide accurate information about the main branch, the tilting pattern of celiac trunk, and the length of CHA prior to the interventional procedure. Gadoliniumenhanced 3D MR angiography is useful for the planning of arterial catheterization of hepatic tumor.

\section{ACKNOWLEDGMENTS}

The authors thank M. Suzuki, RT, M. Yamada, RT, N. Ohya, MD and H. Morita, MD for their generous assistance.

\section{REFERENCES}

1) Dumoulin CL, Hart HR Jr. Magnetic Resonance angiography. Radiology 1986; 161 : 717-720. 
2) Snidow JJ, Aisen AM, Harris VJ, et al. Iliac artery MR angiography: comparison of threedimensional gadolinium-enhanced and twodimensional time-of-flight techniques. Radiology 1995 ; 196 : 371-378.

3) Prince MR, Yucel EK, Kaufmann JA, et al. Dynamic gadolinium-enhanced three dimensional abdominal MR angiography. JMRI 1993; 3 : 877-881.

4) Prince MR. Gadolinium-enhanced MR aortography. Radiology 1994 ; 191 : 155-164.

5) Prince MR, Narasimhan DL, Jacoby WT, et al. Three-dimensional gadolinium enhanced MR angiography of the thoracic aorta. AJR 1996; 166 : 1387-1397.

6) Snidow JJ, Aisen AM, Harris VJ, et al. Threedimensional gadolinium-enhanced MR angiography for aortoiliac inflow assessment plus renal artery screening in a single breath hold. Radiology $1996 ; 198$ : 725-732.

7) Meaney JFM, Prince MR, Nostrant TT, et al. Gadolinium-enhanced MR angiography of visceral arteries in patients with suspected chronic mesenteric ischemia. JMRI 1997; 7 : 171-176.

8) Mueller MF, Siewert B, Strokes KR, et al. MR angiographic guidance for transjugular intrahepatic portosystemic shunt procedures. JMRI 1994 ; 4 : 145-150.

9) Cohen J. A coefficient of agreement for nominal scales. Educ Psychol Meas 1960; 20 : 37-46.

10) Cohen J. Weighted kappa : nominal scale agreement with provision for scaled disagreement or partial credit. Psychol Bull 1968; 70 : 213-230.

11) Landis JR, Koch GG. The measurement of observer agreement for categorical data. Biometrics 1977; 33 : 159-174.

12) Soulen MC. Chemoembolization of hepatic malignancies. Oncology 1994; 8 : 77-84.

13) Matsui O, Kadoya M, Yoshikawa J. et al. Small hepatocellular carcinoma : treatment with subsegmental transcatheter arterial embolization. Radiology $1993 ; 188: 79-83$.

14) Inaba $Y$, Arai $Y$, Sone $Y$, et al. Arterial redistribution to prevent hepatic arterial occlusion and gastric ulcer using hepatic arterial infusion chemotherapy with percutaneous catheterization. GanTo-Kagaku-Ryoho 1992; 19 : 1564-1567.

15) Arai $Y$. Interventional radiology for hepatic arterial infusion chemotherapy. Gan-To-KagakuRyoho 1996; 23 : 1385-1389.

16) Arai $Y$. The investigational strategy and present status of hepatic arterial infusion chemotherapy for liver metastasis for colorectal cancer. Gan-ToKagaku-Ryoho 1993 ; 20 : 2467-2471.

17) Chuang VP, Soo CS, Carrasco CB, Wallace S. Superselective catheterization technique in hepatic angiography. AJR 1983; 141 : 803-811. 